\title{
Circular Antenna Array Synthesis Using Salp Swarm Optimization
}

\author{
A. DURMUŞ
}

\begin{abstract}
Salp Swarm Algorithm (SSA) is used to design an optimal non uniform circular antenna arrays. Salp Swarm Algorithm which mimics the swarming behavior of salps in oceans is a nature-inspired optimization method. As it is simple and easy to apply, it has been applied to many different problems in the literature. SSA method optimally determined the positions and amplitudes of the circular antenna array elements to obtain radiation patterns with a low maximum sidelobe level (MSL) and narrow half power beam width (HPBW). Different sizes of antennas with 8,10 and 12 elements are discussed to demonstrate the capability of the SSA algorithm. The results of MSL and HPB W obtained by SSA for the synthesis of circular antenna arrays are better than other compared optimization methods.
\end{abstract}

Index Terms - Salp swarm algorithm, synthesis of antenna array, circular arrays, maximum sidelobe levels.

\section{INTRODUCTION}

I $\mathrm{N}$ RECENT years, the interest in antenna arrays with highdirectionality and gain used in different areas such as wireless communication, mobile, sonar, radar has been increasing. The communication systems performance used in many different applications is highly dependent on the design of the antenna array used. Antenna arrays can be in different structures such as circular, linear and elliptic depending on their geometric structure. Circular Antenna Arrays (CAAs) among these antenna types have become very popular especially in radar, wireless and mobile communication systems. Unlike linear antenna arrays, circular antenna arrays cover all $360^{\circ}$ of azimuth coverage [1]. CAAs can scan the main beam in any direction without a major change. Also, CAAs are not affected by the mutual effect because they do not have edge elements [2]. Due to these advantages, the optimum design of CAAs is of great interest in the literature. There has been a lot of research in the last decade on CAAs synthesis, an important computational electromagnetic problem. In [3], a genetic algorithm is used for sidelobes reduction of circular antenna arrays by amplitude and position control. Shihab et al. proposed a particle swarm optimization method for design of CAAs with low sidelobes level [4]. A firefly algorithm is applied for circular array optimization in [5]. In [6], seeker optimization algorithm used for designing of circular and concentric circular antenna array for sidelobe reduction. Babayigit et.al. implemented Taguchi's optimization method to the synthesis of circular antenna arrays [7]. Hamza et.al. proposed a genetic algorithm to perform the beam steering and null placement in circular arrays [8]. In [9], moth flame optimization used for designing of linear and circular antenna array for sidelobe reduction. Jamunaa et.al proposed a symbiotic organisms search optimisation algorithm to design of a reconfigurable concentric circular array with phase-only controls differentiating the beams [10]. In [11] Multiverse Optimizer (MVO) and modified MVO are used to perform circular antenna array synthesis. Das et.al. proposed gray wolf optimization and particle swarm optimization with a distribution based update mechanism to design of circular arrays [12]. In [13], Electric Charged Particles Optimization is applied to optimal design of circular antenna array for sidelobe level reduction.

In this paper, Salp Swarm Algorithm (SSA) is used to the synthesis of optimum circular antenna arrays. SSA which mimics the swarming behavior of salps in oceans is a natureinspired optimization method [14]. Since SSA method is an upto-date optimization technique, there are different studies for SSA in the literature in recent years [14-19]. The amplitudes of the circular antenna array elements are determined by SSA in order to obtain optimum radiation pattern with maximum sidelobe level (MSL) reduction and narrow half power beam width (HPBW). The problem of antenna array synthesis consist of HPBW and MSL constraints. SSA has achieved very good results that meet the constraints. HPBW and MSL values of the array patterns can be easily controlled by using SSA. The results obtained with SSA are compared with the results of other well-known optimization methods. SSA demonstrates better performance to synthesizing circular antenna arrays than the other compared optimization techniques.

The rest of the paper is organized as follows: The problem formulation for circular antenna design is examined in Section 2. In Section 3, the SSA method is explained. Section 4 presents numerical results and conclusions are presented in Section 5. 


\section{PRoblem Formulation For Circular ANTENNA DESIGN}

The circular antenna array with $\mathrm{M}$ elements is illustrated in Fig. 1. The antenna array elements are non-uniformly placed in a circle with radius a in the $x-y$ plane In Fig. 1.

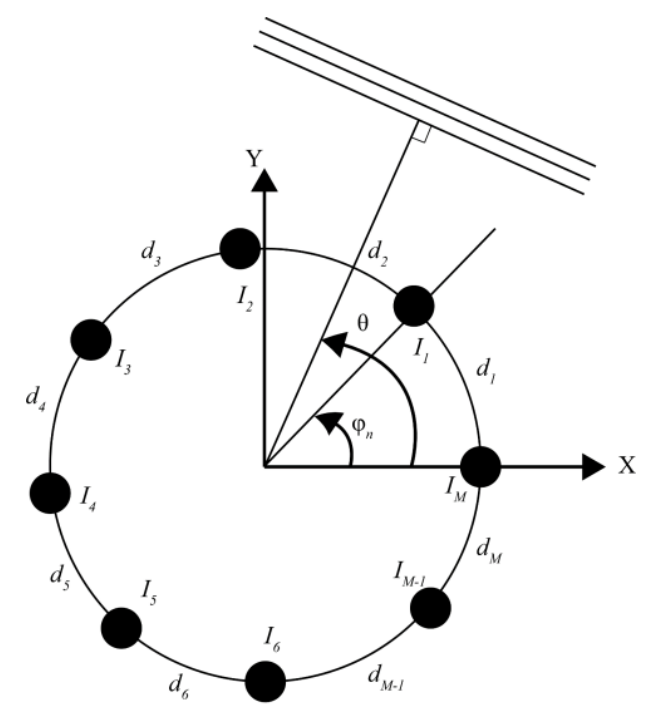

Fig.1: M-elements circular antenna array

Circular antenna array elements are assumed to be isotropic sources with similar properties. The circular antenna array factor is given by [1].

$$
\begin{gathered}
A F(\theta)=\sum_{n=1}^{M} I_{n} \cdot e^{\left(j \cdot k a \cdot \cos \left(\theta-\varphi_{n}\right)+\alpha_{n}\right)} \\
k a=\sum_{i=1}^{M} d_{i} \\
\varphi_{n}=\left(2 \pi \cdot \sum_{i=1}^{n} d_{i}\right) / k a \\
\alpha_{n}=-k a \cdot \cos \left(\theta_{0}-\varphi_{n}\right)
\end{gathered}
$$

where $\alpha_{n}$ and $I_{n}$ are the phase and amplitude values of the nth array element, respectively. The distance between the two antenna arrays elements is indicated by $d_{i} . k$ is the number of wave. The angular position of the $\mathrm{n}$-th array element in $\mathrm{x}-\mathrm{y}$ plane is denoted by $\varphi_{n}$.

The cost function given in Equation 5 is used to designing circular antenna arrays with the decreased HPBW and low MSL values.

$$
T_{\text {cost }}=W_{S L L} \cdot T_{S L L}+W_{H P B W} \cdot T_{H P B W}
$$

where $W_{S L L}$ and $W_{H P B W}$ are the weight factors. $T_{S L L}$ and $T_{H P B W}$ are the functions used for suppressing the MSL and decreasing HPBW values respectively. The function of $T_{S L L}$ can be formulated as follows,

$$
T_{S L L}=\int_{-\pi}^{\varphi_{n 1}} \beta_{S L L}(\varphi) \cdot d \varphi+\int_{\varphi_{n 2}}^{\pi} \beta_{S L L}(\varphi) \cdot d \varphi
$$

where $\varphi_{n 1}$ and $\varphi_{n 2}$ are the two angles at the first nulls on each side of the main beam. $\beta_{S L L}(\varphi)$ can be given as follows,

$$
\beta_{S L L}(\varphi)= \begin{cases}\gamma_{0}(\varphi)-S L L_{d}, & \gamma_{0}(\varphi)>S L L_{d} \\ 0, & \text { elsewhere }\end{cases}
$$

where $S L L_{d}$ is the desired value of MSL. $\gamma_{0}(\varphi)$ is the array factor in $\mathrm{dB}$. The function $T_{H P B W}$ can be formulated by

$$
T_{H P B W}=\left\{\begin{array}{cc}
T_{o}-T_{H P B W \max }, & T_{o}>T_{H P B W \max } \\
0, & \text { elsewhere }
\end{array}\right.
$$

where $T_{H P B W \max }$ and $T_{o}$ are the desired maximum HPBW and the value of HPBW obtained by SSA, respectively.

\section{SALP SWARM ALGORITHM}

The Salp Swarm Algorithm (SSA) is a novel optimization methods proposed by S. Mirjalili, et al. SSA optimization method simulate the swarming behavior of salps in ocean. Salps are living creatures that move by pumping the water in their bodies. The salps move in the depths of the ocean as flocks and these structures are called salp chains. According to the position of the individuals in the salp chain, the salps are divided into two as followers and leaders. The chain of salps is shown in Fig. 2.

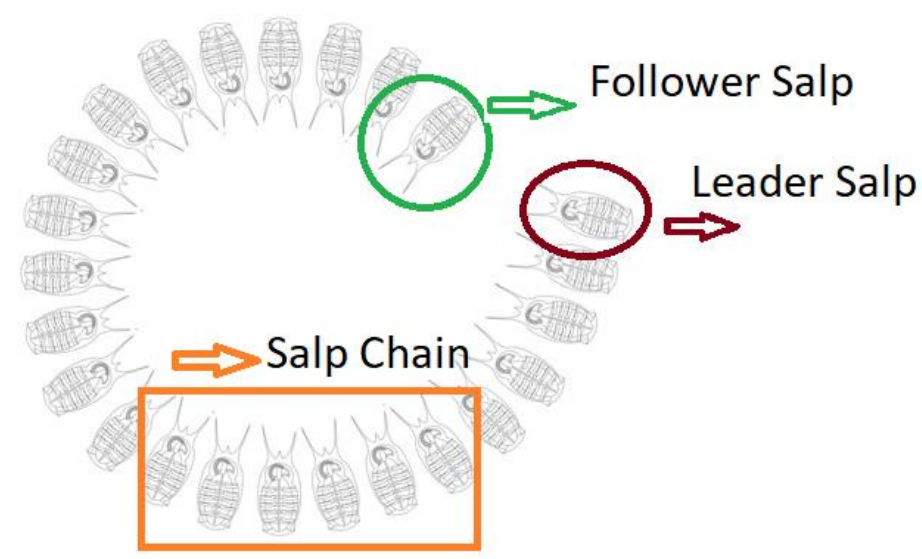

Fig. 2: The chain of salps

The position of each individual in the salps chain is defined in the $n$ dimensional solution space. $n$ is the number of variables to be solved in the optimization problems. The position of leader salp is updated by using Equation 9 [8]. 


$$
k_{i}^{1}= \begin{cases}x_{i}+f_{1}\left(\left(u_{i}-l_{i}\right) f_{2}+l_{i}\right) & f_{3} \geq 0 \\ x_{i}-f_{1}\left(\left(u_{i}-l_{i}\right) f_{2}+l_{i}\right) & f_{3}<0\end{cases}
$$

where $x_{i}$ and $k_{i}^{1}$ are the food position and the first salp position in the $\mathrm{i}$-th dimension respectively. $l_{i}$ and $u_{i}$ corresponds to lower and upper bound of the $\mathrm{i}$-th dimension. The $f_{2}$ and $f_{3}$ coefficients are random numbers generated in the range $[0,1]$. The $f_{1}$ coefficient is calculated from the expression below

$$
f_{1}=2 e^{-\left(\frac{4 d}{D}\right)^{2}}
$$

where $\mathrm{D}$ and $\mathrm{d}$ represent the maximum iterations number and the current iteration number, respectively. The position of the followers are updated by using equation 11 .

$$
k_{i}^{j}=\frac{1}{2}\left(k_{i}^{j}+k_{i}^{j-1}\right)
$$

Detailed information about SSA is given in the reference [8].

\section{NUMERICAL RESULTS}

All simulation studies in this paper were done on a computer with $16 \mathrm{~Gb}$ RAM and $2.6 \mathrm{GHz}$ i7 processor. MATLAB is the software program to implement SSA. The population parameter of SSA is fixed to 40 . The main goal of circular antenna array synthesis problems is to achieve the lowest MSL and narrowest HPBW values to improve radiation pattern quality. Both position and amplitude values of circular antenna array elements are determined using SSA to achieve the desired radiation pattern with low MSL and narrow HPBW values. Circular antenna arrays with 8,10 and 12 elements are examined to demonstrate the flexibility and performance of the SSA method.

In the first example, the amplitudes and positions values of circular antenna array with 8 elements are achieved by SSA. The number of maximum iteration is 1000 . The radiation pattern obtained by SSA is shown in Fig. 3. The pattern achieved by other four optimization algorithms are also given in Fig. 3.

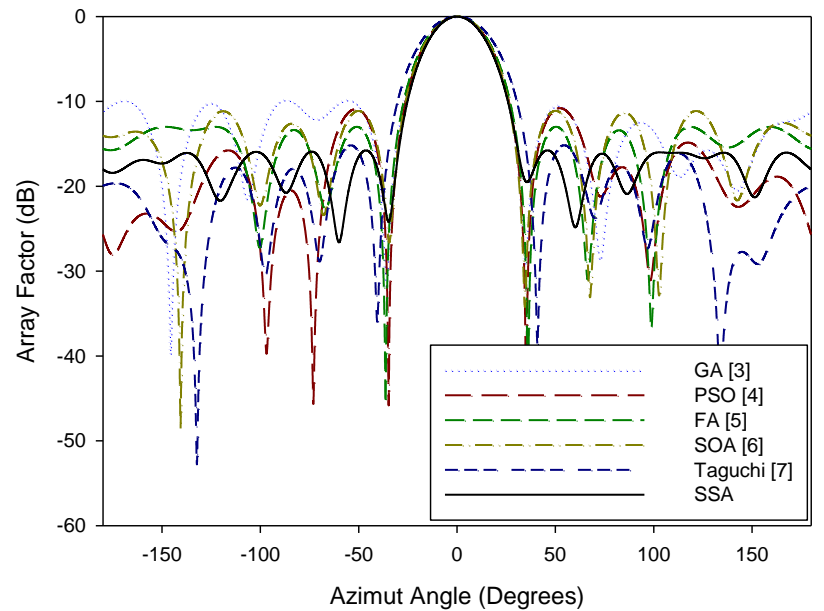

Fig. 3: Radiation pattern of 8-elements circular array.

Table I demonstrates the HPBW and MSL values of the radiation patterns achieved by SSA and other comparison algorithms. According to Fig. 3 and Table I, the MSL and HPBW results obtained by SSA are better than GA [3], PSO [4], FA [5], SOA [6] and Taguchi [7] methods.

TABLE I

COMPARISON OF THE MSL AND HPBW VALUES FOR 8-ELEMENTS CAA DESIGN

\begin{tabular}{ccc}
\hline Methods & MSL (dB) & HPBW (degrees) \\
\hline SSA & -15.78 & 29.90 \\
GA [3] & -9.81 & 32.22 \\
PSO [4] & -10.80 & 31.68 \\
FA [5] & -12.99 & 32.76 \\
SOA [6] & -11.11 & 31.68 \\
Taguchi [7] & -15.17 & 36.72 \\
\hline
\end{tabular}

In the second example, circular antenna arrays with 10 elements are considered. The maximum iteration number of SSA is 1000 . The radiation patterns obtained by SSA, GA [3], PSO [4], FA [5], SOA [6] and Taguchi [7] are shown in Fig. 4.

Along with the values obtained with SSA, the MSL and HPBW values of GA [3], PSO [4], FA [5], SOA [6] and Taguchi [7] methods are listed for comparison in Table II. The MSL value of radiation pattern achieved by SSA is $-15.35 \mathrm{~dB}$. As can be clearly seen from the Figure 4 and Table II, SSA has obtained better MSL value than other optimization methods compared. Also, if we examine the HPBW value, it is seen that the value achieved with SSA is better than the results of Taguchi [7] method. 


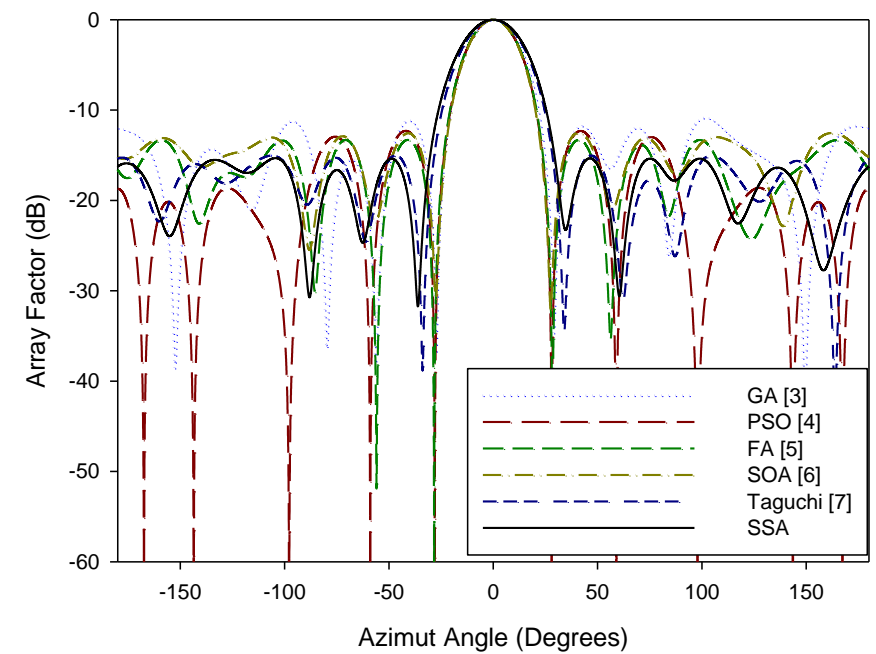

Fig. 4: Radiation pattern of 10-elements circular array.

TABLE II

COMPARISON OF THE MSL AND HPBW VALUES FOR 10-ELEMENTS CAA DESIGN

\begin{tabular}{ccc}
\hline Methods & MSL (dB) & HPBW (degrees) \\
\hline SSA & 15.35 & 29.11 \\
GA [3] & -10.85 & 25.56 \\
PSO [4] & -12.30 & 24.48 \\
FA [5] & -13.30 & 24.84 \\
SOA [6] & -12.50 & 24.12 \\
Taguchi [7] & -15.03 & 29.16 \\
\hline
\end{tabular}

TABLE III

COMPARISON OF THE MSL AND HPBW VALUES FOR 12-ELEMENTS CAA DESIGN

\begin{tabular}{ccc}
\hline Methods & MSL (dB) & HPBW (degrees) \\
\hline SSA & -18.01 & 16.86 \\
GA [3] & -11.80 & 20.52 \\
PSO [4] & -13.67 & 20.88 \\
FA [5] & -14.20 & 20.52 \\
SOA [6] & -14.11 & 20.34 \\
Taguchi [7] & -17.68 & 16.92 \\
\hline
\end{tabular}

In the last example, the SSA technique is used for optimization of the circular antenna array with 12 elements. The number of maximum iteration is has been set to 1000 to find optimum solution. The radiation pattern obtained after optimizing the position and amplitude values of the circular arrays with SSA is shown in Fig. 5. MSL and HPBW values of the radiation patterns are given in Table III comparatively. As can be clearly seen from Table III, the MSL and HPBW values obtained by SSA are better than that obtained by other compared algorithms.

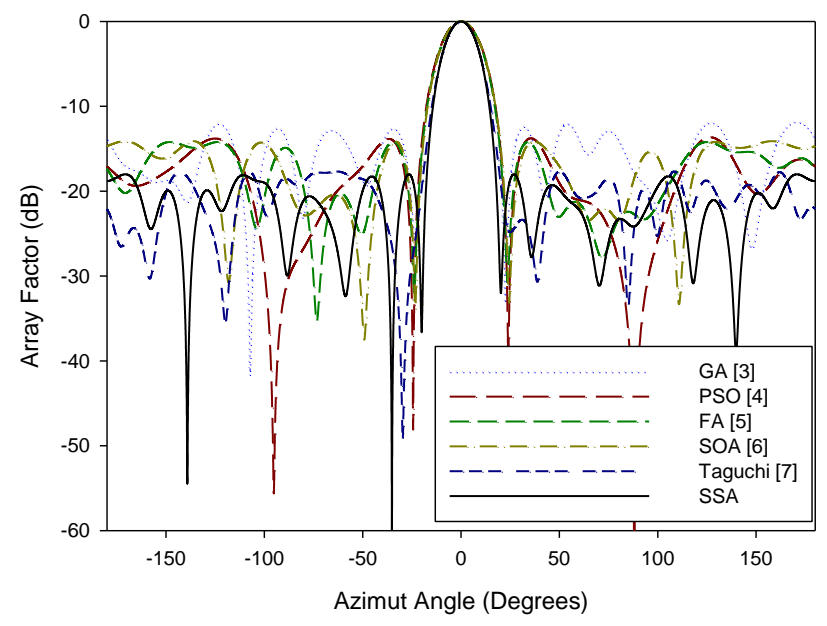

Fig. 5: Radiation pattern of 12-elements circular array.

The convergence curves obtained for 8,10 and 12 element circular antenna arrays design are given in Fig. 6.

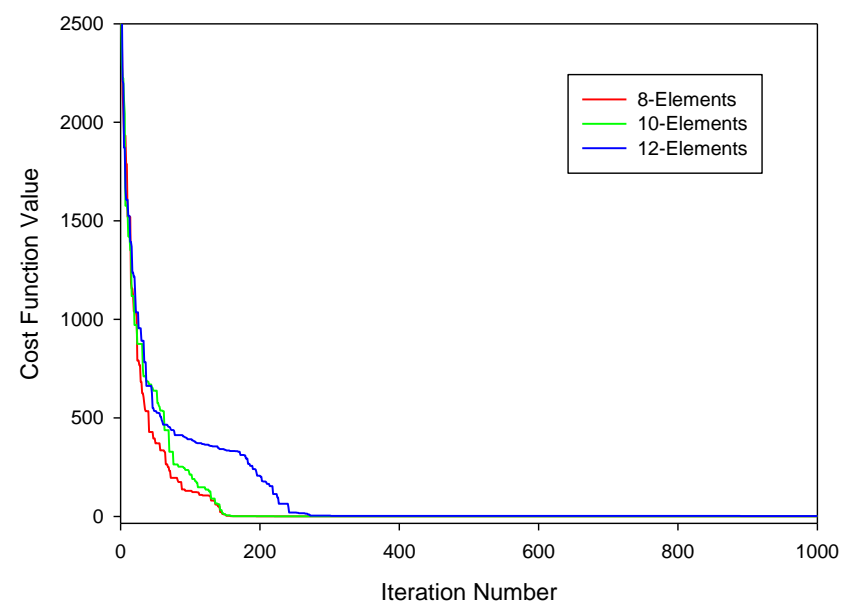

Fig. 6: The convergence curve of CAA with 8-elements, 10-elements and 12-elements.

Table IV tabulates the amplitudes and positions values of the circular antenna array with 8,10 , and 12elements.

\section{CONCLUSION}

In this study, the position and amplitude values of the nonuniform circular antenna arrays having 8, 12, and 20 isotropic elements are optimized by SSA method. The radiation pattern with low MSL and narrow HPBW values are obtained with the proposed SSA algorithm. The results show that SSA is successful in achieving radiation pattern with low MSL and narrow HPBW for circular antenna array synthesis. Generally, the MSL and HPBW values obtained with SSA are better than the other compared algorithms. In addition, by making changes in the algorithm and creating hybrid structures, better results can be obtained in later studies. 
TABLE IV

THE AMPLITUDE AND POSITION VALUES OF NON-UNIFORM CIRCULAR ARRAY WITH 8-ELEMENTS, 10-ELEMENTS, AND 12-ELEMENTS

\begin{tabular}{|c|c|c|}
\hline 8-Elements (Fig. 3) & {$\left[\begin{array}{llllllll}0.9369 & 0.3191 & 0.3704 & 0.6591 & 0.8065 & 0.3922 & 1.0000 & 0.4209\end{array}\right]$} & {$\left[\begin{array}{llllllll}0.3710 & 1.0107 & 0.3507 & 0.9704 & 0.5172 & 0.9543 & 1.4366 & 0.3050\end{array}\right]$} \\
\hline 10-Elements (Fig. 4) & 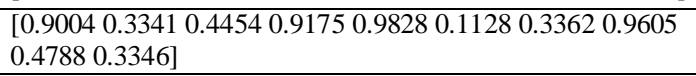 & 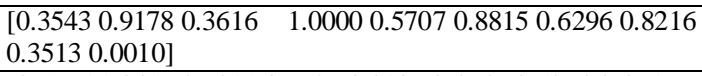 \\
\hline 12-Elements (Fig. 5) & 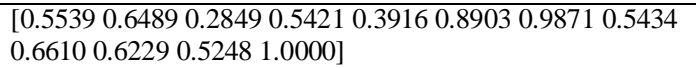 & 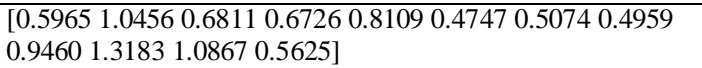 \\
\hline
\end{tabular}

\section{REFERENCES}

[1] C. A. Balanis, Antenna theory: analysis and design, 3rd edn. Wiley, New York, 2005.

[2] J. R. Mailloux, Phased array antenna handbook. Artech House, Boston, 2005.

[3] M. A. Panduro, A. L. Mendez, R. Dominguez, G. Romero. "Design of non-uniform circular antenna arrays for side lobe reduction using the method of genetic algorithms." AEU Int. J. Electron Commun., vol.60.7, 2006, pp. 713-717.

[4] M. Shihab, Y. Najjar, N. Khodier. "Design of non-uniform circular antenna arrays using particle swarm optimization." J. Electr. Eng., vol. 59, 2008, pp. 216-220.

[5] A. Sharaqa, N. Dib. "Circular antenna array synthesis using firefly algorithm." Int. J. RF Microw. Comput. Eng., vol.24, 2014, pp. 139-146.

[6] K. Guney, S. Basbug. "A parallel implementation of seeker optimization algorithm for designing circular and concentric circular antenna arrays." Appl. Soft Comput. vol.22, 2014, pp. 287-296.

[7] B. Babayigit, E. Senyigit. "Design optimization of circular antenna arrays using Taguchi method." Neural Computing \& Application, vol. 28, 2017, pp.1443-1452.

[8] A. Hamza, H. Attia, "Fast Beam Steering and Null Placement in an Adaptive Circular Antenna Array." IEEE Antennas and Wireless Propagation Letters, vol. 19, 2020, pp. 1561-1565.

[9] A. Das, D. Mandal, S.P. Ghoshal, R. Kar. "Moth flame optimization based design of linear and circular antenna array for side lobe reduction." International Journal of Numerical Modelling: Electronic Networks, Devices and Fields, vol. 32, 2019, pp.1-15.

[10] D. Jamunaa, F. N. Hasoon, G. K. Mahanti. "Symbiotic organisms search optimisation algorithm for synthesis of phase-only reconfigurable concentric circular antenna array with uniform amplitude distribution." International Journal of Electronics Letters, 2019, pp. 1-12.

[11] A. E. Taser, K. Guney, E. Kurt. "Circular Antenna Array Synthesis Using Multiverse Optimizer." International Journal of Antennas and Propagation, 2020.

[12] A. Das, D. Mandal, R. Kar. "An optimal circular antenna array design considering mutual coupling using heuristic approaches." International Journal of RF and Microwave Computer-Aided Engineering, 2020, pp. 114.

[13] H. R. E. H Bouchekara. "Electric Charged Particles Optimization and its application to the optimal design of a circular antenna array." Artificial Intelligence Review, 2020, pp. 1-36.

[14] S. Mirjalili, A. H. Gandomi, S. Z. Mirjalili, S. Saremi, H. Faris, S. M. Mirjalili. "Salp Swarm Algorithm: A bio-inspired optimizer for engineering design problems." Advances in Engineering Software, vol. 114, 2017, pp. 163-191.

[15] H. Faris, M. Mafarja, A. Heidari, I. Aljarah, A. Al-Zoubi, S. Mirjalili, et al. "An efficient binary salp swarm algorithm with crossover scheme for feature selection problems. " Knowledge-Based Systems, vol. 154, 2018, pp. 43-67.

[16] R. Abbassi, A. Abbassi, A. Heidari, S. Mirjalili. "An efficient salp swarminspired algorithm for parameters identification of photovoltaic cell models." Energy Conversion and Management, vol. 179, 2019, pp. 362 372.

[17] E. E. Elattar, S. K. ElSayed. "Probabilistic energy management with emission of renewable micro-grids including storage devices based on efficient salp swarm algorithm." Renewable Energy, vol. 153, 2020, pp. 23-35.

[18] X. Zhao, P. Wu, X. Yin. "A quadratic penalty item optimal variational mode decomposition method based on single-objective salp swarm algorithm." Mechanical Systems and Signal Processing, vol. 138, 2020, p. 106567.

[19] W. Liu, R. Wang, J. Su. "An Image Impulsive Noise Denoising Method Based on Salp Swarm Algorithm." International Journal of Education and Management Engineering, vol. 10, 2020, p. 43.

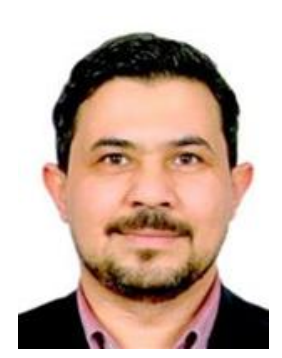

Ali DURMUŞ was born in Kayseri, Turkey in 1980. He received the B.Sc., M.Sc. and Ph.D. degrees, all in Electrical and Electronics Engineering, from Erciyes University, Kayseri Turkey in 2003, 2005, and 2016 respectively. He served as a lecturer at the Department of Electricity and Energy, Erciyes University from 2010 to 2018. Currently he is an assistant professor at the Department of Electricity and Energy, Kayseri University. His research interests are antennas, antenna arrays, meta-heuristic algorithms, and computational electromagnetics. 